\title{
Long-term impact of ten-valent pneumococcal conjugate vaccine (PCV10) on pneumonia among vaccine-eligible children in Finland
}

Arto A. Palmu', Hanna Rinta-Kokko², Hanna Nohynek ${ }^{3}$, J. Pekka Nuorti³, Jukka Jokinen².

National Institute for Health and Welfare, the Department of Public Health Solutions, Tampere ${ }^{1}$ and Helsinki ${ }^{2}$, and the Department of Health Security ${ }^{3}$, Helsinki, Finland.

Background

- PCV10 was introduced into the Finnish National Vaccination Programme (NVP) in Sep-2010 using a $2+1$ schedule with estimated uptake at $93 \%$.

- We evaluated the long-term impact of PCV10 on pneumonia among vaccine-eligible children during the first six years after the NVP introduction.

Methods: cohort comparison

- The target cohort eligible for PCV10-NVP born 06/201009/2016 was compared with a calendar-time and agematched (3-78 months) reference cohort before NVP introduction (Figure 1).

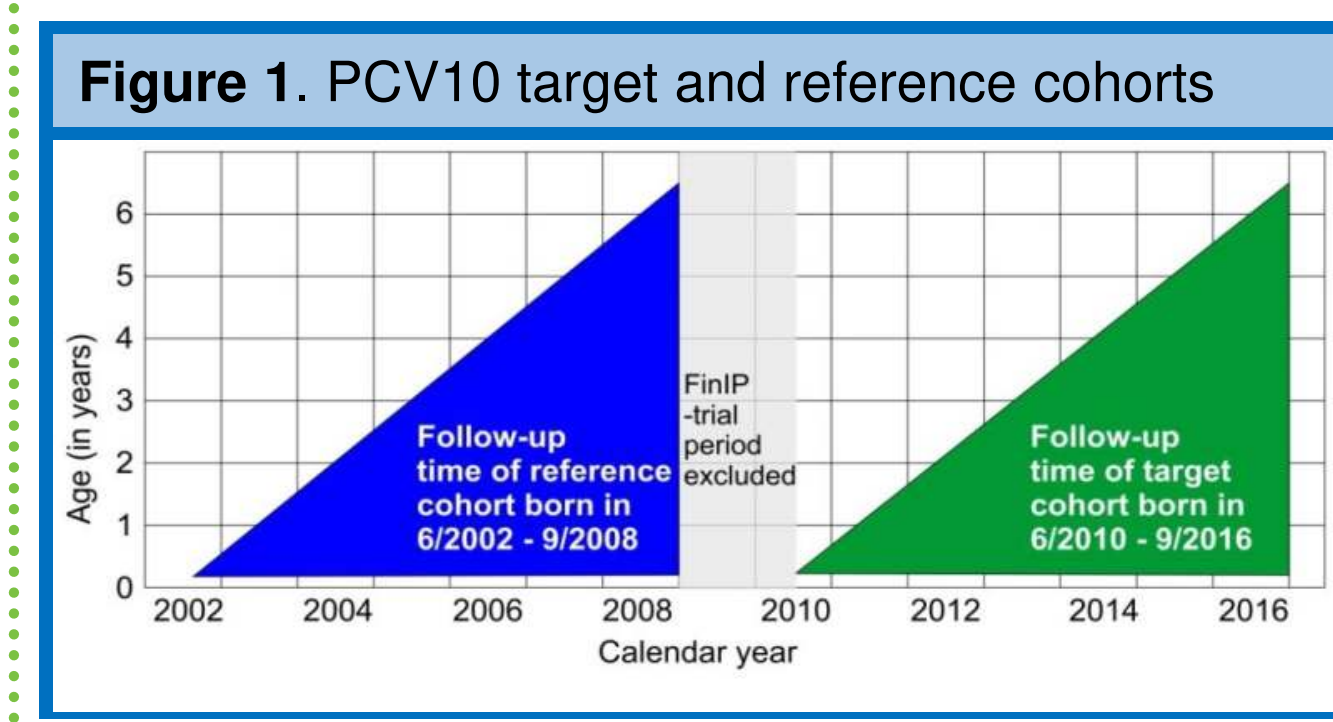

Figure 3. Annual incidences of empyema/abscess

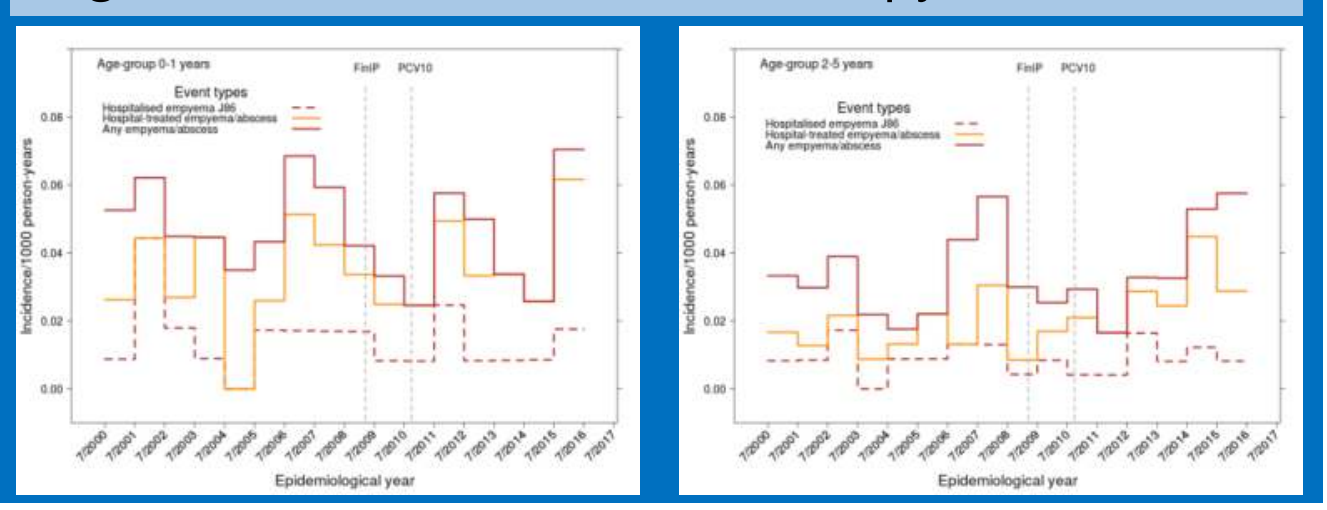

Figure 2. Pneumonia episodes in children $<2$ years and 2-5 years by epidemiologic year, Finland 2000-2017
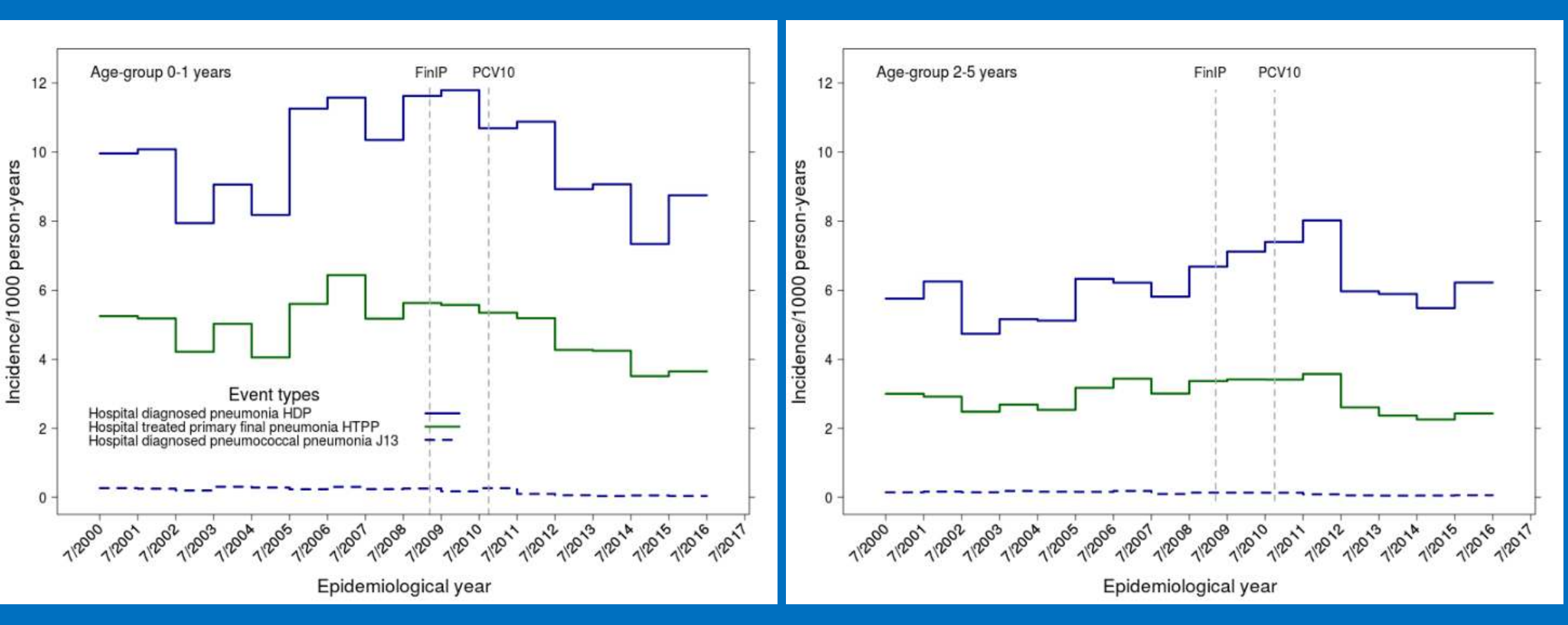

Table. Pneumonia incidences with relative and absolute rate reductions after vaccine introduction.
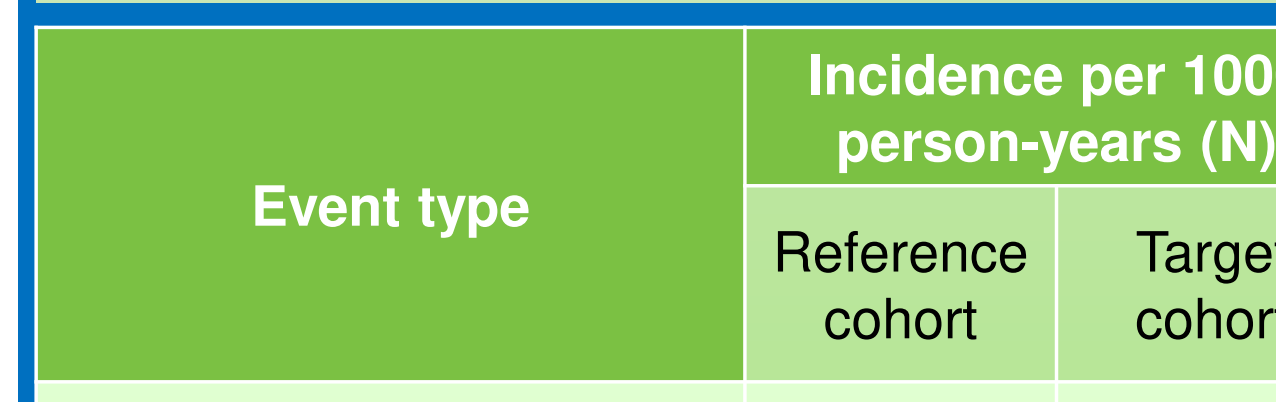

Hospital-diagnosed pneumonia, HDP 8.3

Hospital-treated primary pneumonia, HTPP HDP, pneumococcal (ICD-10 J13) Any empyema/abscess, (ICD-10 J85/J86)

Hospitalization for

empyema/abscess

8.3
$(10085)$

4.3

4.3
$(5215)$

(5215)

0.22
$(266)$

$(266)$
0.05

(58)

0.03

(33)

7.8
$(9640)$

3.3

(4067)

0.06

(69)

$(69)$
0.05

0.05

(64)

0.04

(48)
Rate reduction ( $R R$ ) in target vs. reference cohort

Relative RR, Absolute RR, per 1000 $\%(95 \% \mathrm{Cl}) \quad$ person-years $(95 \% \mathrm{Cl})$

$\begin{array}{cc}7 & 0.6\end{array}$

$(4,9) \quad(0.4,0.8)$

\begin{tabular}{l|r}
24 & 1.0
\end{tabular}

$(21,27)$

75

$(67,81)$

$-7.5$

$(-54,25)$

$-42$

$(-123,9)$

\section{Methods: Case definitions}

Hospital in- and outpatient discharge notifications with diagnoses compatible with any hospital-diagnosed pneumonia (HDP, ICD-10 codes J10.0/J11.0/J12$\mathrm{J} 18 / \mathrm{J} 85.1 / \mathrm{J} 86)$ or any empyema/lung abscess (ICD-10 codes J85/J86) were collected from the national Care Register.

We calculated rates of HDP and hospital-treated primary pneumonia (HTPP, pneumonia as the primary diagnosis after inpatient hospitalization) and empyema before and after NVP introduction

- No radiological evaluation data were available.

Episode duration was 90 days.

\section{Results}

- Relative rate reduction in HDP was $7 \%$ and in HTPP 24\%; absolute reduction for HTPP was 1/1000 person-years (Table).

Number of lung abscess/empyema diagnoses fluctuated by years, but remained low with nonsignificant increase (Table, Figure 3).

Conclusions

This nation-wide study provides evidence of the long-term effects of the 10-valent PCV against pneumonia in a routine vaccination program setting Potential increase in empyema diagnoses is inconclusive and requires further follow-up.

- The PCV10 introduction has prevented over 1000 pneumonia hospitalizations during 2010-2016 in the : vaccine-eligible target cohort. 